\title{
Classifying Environmental Monitoring Systems
}

\author{
Mauno Rönkkö ${ }^{1}$, Ville Kotovirta ${ }^{2}$, and Mikko Kolehmainen ${ }^{1}$ \\ 1 Department of Environmental Science, University of Eastern Finland, \\ PO Box 1627, 70211 Kuopio, Finland \\ \{mauno.ronkko, mikko.kolehmainen\}@uef .fi \\ ${ }^{2}$ VTT Technical research Centre of Finland, \\ PO Box 1000, 02044 VTT, Finland \\ ville.kotovirta@vtt.fi
}

\begin{abstract}
In this article the diversity of environmental monitoring systems is studied. The number of such systems is steadily increasing each year, as systems are tailored to specific, growing needs of authorities, corporate users and citizens. Because of this, it becomes harder to compare systems and their functionality. Systems that appear to have the same functionality may turn out to be tailored for different application domains. Likewise, a chosen system may later on turn out to have insufficient support for connectivity and interoperability, although it provides the best support for core functionality requirements. To make sense of the ever growing diversity, and as the main contribution, a method for classification and analysis is proposed. The method is generic to environmental monitoring systems. The use of the method is also illustrated. The classification results yield even for a limited number of systems relevant clusters that help in identifying critical properties for further inspection.
\end{abstract}

Keywords: Environmental information systems, Environmental monitoring, Systems Architecture, Systems Analysis, Systems Classification.

\section{Introduction}

There are currently many factors driving the design and development of both national and international environmental information systems. One of the most notable of these factors is the green house effect. Reducing energy consumption, cutting greenhouse gas emissions and eliminating energy wastage are of global interest. Another significant factor has to do with the vulnerability of modern society in case of natural disasters. This has also been highlighted by recent extreme weather phenomena exhibited around the globe.

Not surprisingly, as pointed out by Messer et al. 11 "High-resolution, continuous, accurate monitoring of the environment is of great importance for many applications from weather forecasting to pollution regulation." Environmental monitoring has, thus, become an attractive topic for state-of-the-art research. For instance, monitoring of the Fukushima incident is still relevant for impact assessment as discussed by Cyranoski et al. 2]. Similarly, continuous monitoring of factories and power plants is critical as they may cause long term effects 3 .

J. Hřebíček et al. (Eds.): ISESS 2013, IFIP AICT 413, pp. 533-542, 2013.

(C) IFIP International Federation for Information Processing 2013 
As the number of new environmental monitoring systems grows, it becomes harder to understand the similarities and differences between the systems. In particular, it becomes harder to understand what the differences mean in terms of future applicability and interoperability. Without this critical information, there is a temptation to develop a new system rather than to adapt and use an existing one; thus, making the overall problem worse.

To make sense of the ever growing number of environmental monitoring systems, a method for the classification is proposed in this article. The method identifies three strategic viewpoints: application domain, functionality, and architecture. The proposed method is used in this article to classify a selected set of environmental monitoring systems. Even for a limited case, the results identify some relevant and interesting clusters. Clustering reveals clearly, which properties are shared by the systems and which properties differentiate a specific system from the rest of the systems within a cluster.

This article is organized as follows. In Section 2 we present the proposed classification method. In Section 3 we describe the environmental monitoring systems considered for classification, and present the classification results. Finally in Section 4 follows the conclusion.

\section{Proposed Classification Method}

There are three strategic viewpoints that are necessary for classification: application domain, functionality, and architecture. The application domain viewpoint describes not only the purpose and need for the use of the system, but also the domain of interest. The functionality viewpoint describes what the system does to satisfy the needs for its use. The architecture viewpoint describes how the system is implemented to provide its functionality. These viewpoints are intentionally orthogonal, to support the analysis process. However, even for these three viewpoints, there are many properties of interest. Therefore, the analysis is limited here to specific properties per viewpoint, as described next.

Application domain viewpoint emphasizes the purpose and the need for the system, including the domain of interest. From this viewpoint there are four kinds of properties of interest:

1. Phenomenon: What is the monitored environmental phenomenon?

2. Interest: Why the end-user is interested in the phenomenon?

3. Task: What is the system used for?

4. Benefit: How do the end-users benefit from using the system?

Functionality viewpoint emphasizes the activities of the system that satisfy the end-user's need. From this viewpoint, there are six properties of interest:

1. Operation: What operation is performed by the system?

2. Interaction: How is the operation initiated and carried out?

3. Performance: What kind of performance is expected from the operation?

4. Reliability: How dependable the operation is expected to be? 
5. Methods: What methods are used to perform the operation?

6. Maintenance: What kind of maintenance is expected?

Architecture viewpoint emphasizes the details of the system that realize the activities. From this viewpoint, there are four properties of interest:

1. Implementation: How is the system implemented?

2. Data: What kind of data is used by the system?

3. Capacity: What kind of performance can the system provide?

4. Connectivity: How is the system connected to other systems?

The actual analysis is performed by cross-tabulation over the properties for each viewpoint, where each cell indicates if the considered system supports the combined properties. This results in three tables as shown in Figures [1, 3, and 5. Consequently, the tables provide a qualitative analysis, a comparison over the considered systems. The tables show three different kinds of clusters: properties that are unique to some system, properties that are shared among a few systems, and properties that are common to most of the systems. Properties that are unique to a system indicate the competitive edge of the system. Properties that are shared among a few systems indicate a higher level feature that is somehow central to those systems. Lastly, properties that are common to most of the systems indicate compulsory requirements. Thus, meaningful clusters are found by finding clusters of properties that are shared by only a few systems.

\section{Results}

In this section, environmental monitoring systems that were classified are introduced first shortly. A set of systems were chosen to represent different kinds of environmental monitoring systems. The classification results and discussion are presented at the end of this section.

\subsection{Environmental Monitoring Systems to Be Classified}

Measuring and Forecasting. For this class of systems, NOAA Integrated Ocean Observing Systems (IOOS) was considered. As presented by Harlan et al. 4], the NOAA IOOS uses a high frequency radar network for measuring ocean surface currents in United States. It consists of more than 100 high frequency radars. As pointed out by Harlan et al., the radar network constitutes the largest set of ocean current observations in existence.

Integrated Service. For this class of systems, a weather service application integrating Weather Underground and INTAMAP [5] was considered, later called INTAMAP WU. As presented by Williams et al. [6], the application provides quality control for weather monitoring over a collective network of privately owned weather stations. It provides an OGC standard Observations \& Measurements (O\&M) interface and uses UncertML [7] for describing uncertainties for the Weather Underground data.

Household Monitoring. For this class of systems, a system for measuring and monitoring indoor air quality and energy efficiency, AsTEKa, by Skön et al. 
8] was considered. It consists of a sensor network for measurements and data capture, a database for data storage, and an application server for monitoring services and support for external user interface built on top of a web browser. The sensor network gathers data for instance about humidity, temperature, and carbon dioxide, as well as data about water and electricity consumption.

Environmental Safety and Security. For this class of systems, a distributed service network for security applications, TiTiMaKe [9], was considered. According to Rönkkö et al., TiTiMaKe integrates heterogeneous services, including a dedicated sensor network, vehicle detection and classification service, reachability computation and analysis service, chemical dispersion computation and modeling service, and a spatio-temporal population modeling service. TiTiMaKe implements safety and security applications for traffic monitoring, and estimating affected people and risk groups in case of a chemical accident.

Navigation and Planning. For this class of systems, Icebreaker Plot (IBPlott) [10] was considered. IBPlott is a system for icebreaker navigation and assistance planning. It delivers near real-time ice, weather, and hydrological data to users making routing decision in dynamic environmental conditions. The system takes satellite images, weather observations and forecasts, water level observations and forecasts, ice charts and ice model forecasts, as well as ship traffic information as an input.

Participatory Sensing. For this class of systems, EnviObserver [1] was considered. EnviObserver uses people as sensors in collecting observations about the environment. Users use a specialized mobile application to report environmental phenomena and the observations. Time of the observations and the location of the users are stored in the server for later analyses. The observations collected can be viewed graphically near real-time, and alerts can be generated.

\subsection{Classification Results for Application Domain}

The cross-tabulation over application domain properties are presented in Figure 2. There are four distinct clusters of systems. The clusters are health and comfort, sea and water, improved security, and improved efficiency.

Health and Comfort. INTAMAP WU, AsTEKa, and EnviObserver forms the first cluster. The shared properties have to do with measuring, learning, and analysis related to comfort, health, and well-being for citizens and households. There is also a strong emphasis on delivering personalized information to citizens.

Sea and Water. NOOA IOOS HFR, IBPlott, and EnviObserver form the second cluster. This cluster targets the services for authorities for monitoring and decision making. These systems share strictly the interest of water, sea or lake, as the application domain.

Improved Security. OOA IOOS HFR, TiTiMaKe, IBPlott, and EnviObserver form the third cluster. This cluster subsumes the cluster for sea and water; however, these systems share strongly the common interest for improved security by 


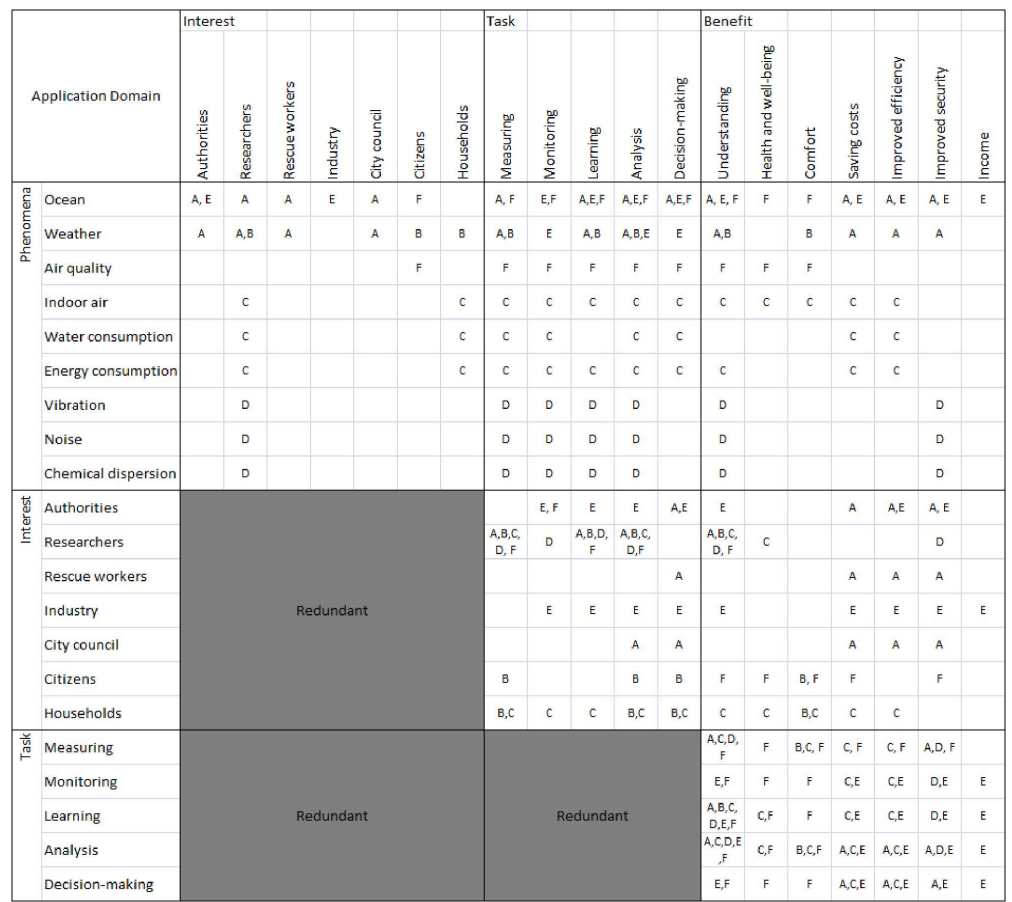

Fig. 1. Cross-tabulation of the properties for application domain. Labeling: $A=$ NOOA IOOS, B=INTAMAP WU, C=AsTEKa, D=TiTiMaKe, E=IBPlott, $\mathrm{F}=$ EnviObserver
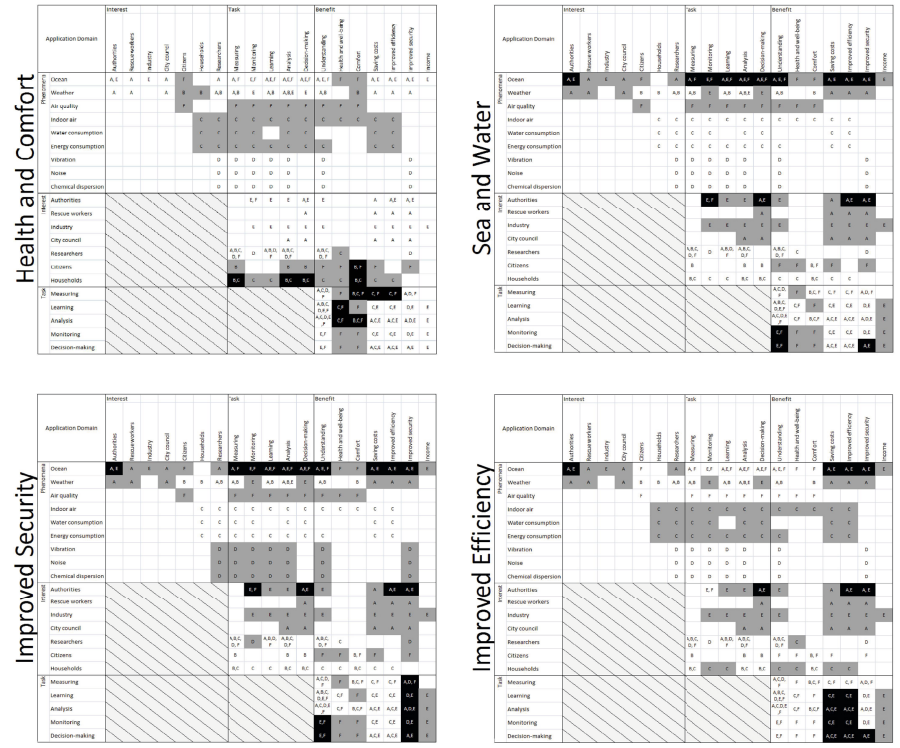

Fig. 2. Clusters found for the application domain viewpoint, Figure 1 the shared properties are indicated with black fill and the disjoint properties with gray fill 
targeting the services for authorities. There is also a shared interest for understanding the underlying phenomena.

Improved Efficiency. OOA IOOS HFR, AsTEKa, and IBPlott form the last cluster. These systems show a strong emphasis on saving costs. Interestingly the tasks involve not only monitoring and decision making, but also learning and analysis.

\subsection{Classification Results for Functionality}

The cross-tabulation over functionality properties are presented in Figure 4. In the figure, properties unique to the clustered systems are indicated with yellow, and shared properties are indicated with red. There are three distinct clusters of systems. The clusters are local-monitoring, decision making, and robust together with reliable.

Local Monitoring. AsTEKa and TiTiMaKe form the first cluster. Both systems perform monitoring of present data with model-based methods. These systems do, however, differ in application domain, which is reflected in the operational properties.

Decision Making. NOOA IOOS HFR, INTAMAP WU and TiTiMaKe form the second cluster. In this cluster, shared operations are: producing new data, forecasting, extracting knowledge, and delayed response. The systems differ on data flow and locality of measurements. Also, NOOA IOOS HFR and TiTiMaKe have maintenance that INTAMAP WU does not really have.

Robust and Reliable. NOOA IOOS HFR, IBPlott, and EnviObser form the third cluster. The cluster is not cohesive, as only IBPlott and EnviObserver have a subscription service for real-time phenomena monitoring. Moreover, NOOA IOOS HFR and IBPlott have uncertainty management, but only NOOA IOOS HFR and EnviObserver use statistical methods.

\subsection{Classification Results for Architecture}

The cross-tabulation over architecture properties are presented in Figure 6] In the figure, properties unique to the clustered systems are indicated with yellow, and shared properties are indicated with red. There are four distinct clusters of systems. The clusters are open systems, closed systems, large data flow, and externally dependent.

Open Systems. INTAMAP WU and EnviObserver form the first cluster. These systems have loosely coupled, international components and sensors with open interfaces. These two systems differ only by use of programming languages and use of standards.

Closed Systems. NOOA IOOS HFR, AsTEKa, and TiTiMaKe form the second cluster. For these systems, use of standards and locality (or nationality) is 


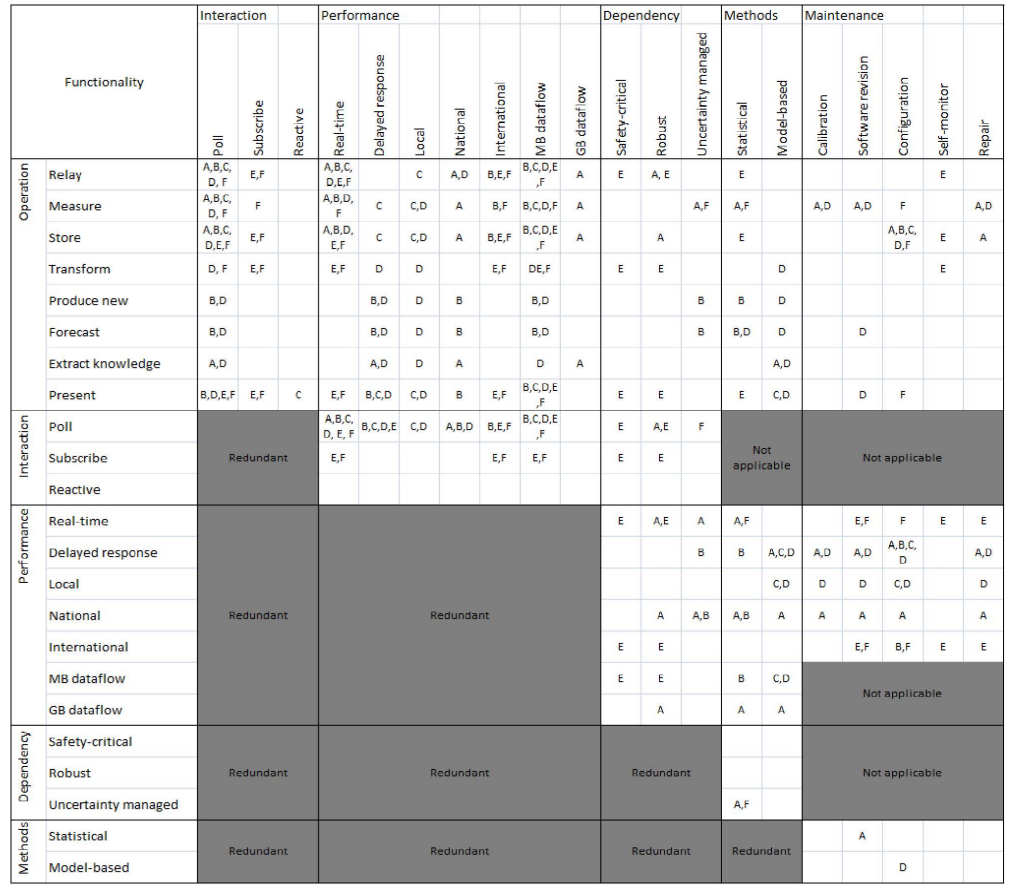

Fig. 3. Cross-tabulation of the properties for functionality. Labeling: $A=$ NOOA IOOS, $\mathrm{B}=\mathrm{INTAMAP}$ WU, $\mathrm{C}=$ AsTEKa, $\mathrm{D}=$ TiTiMaKe, $\mathrm{E}=\mathrm{IBPlott}, \mathrm{F}=$ EnviObserver.
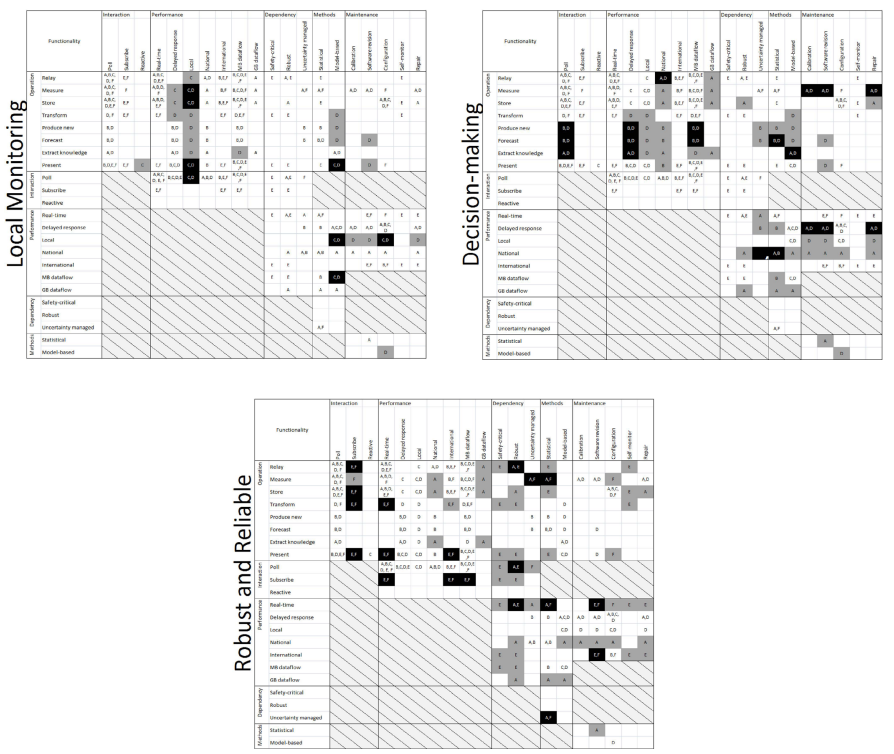

Fig. 4. Clusters found for the functionality viewpoint, Figure 3 the shared properties are indicated with black fill and the disjoint properties with gray fill 


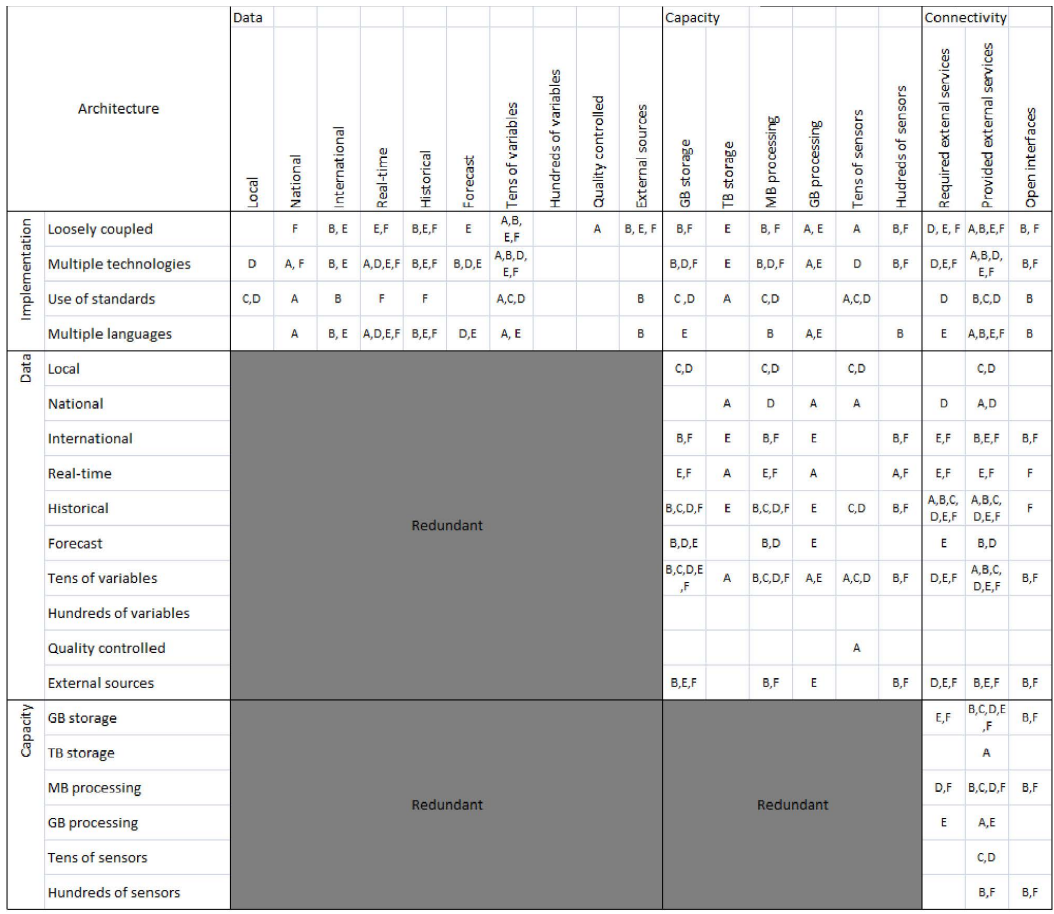

Fig. 5. Cross-tabulation of the properties for architecture. Labeling: $A=$ NOOA IOOS, $\mathrm{B}=\mathrm{INTAMAP}$ WU, $\mathrm{C}=$ AsTEKa, $\mathrm{D}=$ TiTiMaKe, $\mathrm{E}=\mathrm{IBPlott}, \mathrm{F}=$ EnviObserver.
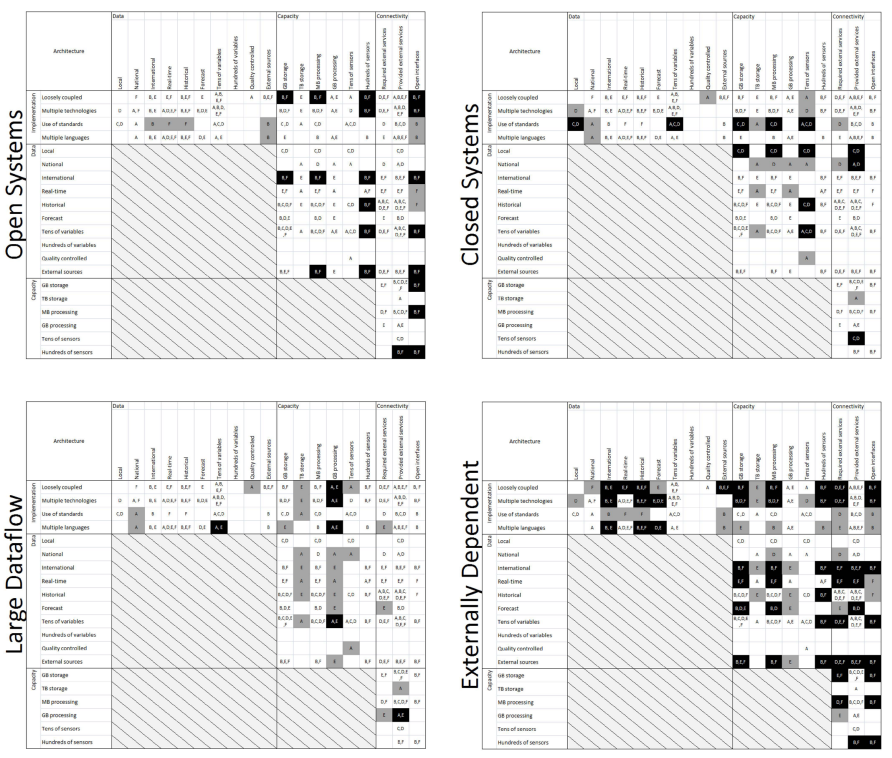

Fig. 6. Clusters found for the architecture viewpoint, Figure 5 , the shared properties are indicated with black fill and the disjoint properties with gray fill 
strongly expressed. In addition, these systems merely provide external interfaces for specific purposes.

Large Data Flow. OOA IOOS HFR, and IBPlott form the third cluster. Interestingly, these systems share partially the same interest domain, ocean. However, as shown in the cluster, these systems have almost nothing else in common than the use of large data flows.

Externally Dependent. INTAMAP WU, TiTiMaKe, IBPlott, and EnviObserver form the last cluster. These systems have loosely coupled components and they also use multiple technologies and programming languages. The cluster is actually divided into to sub-clusters, where IBPlott and EnviObserver focus more on the real-time aspects, and the other two systems focus more on the forecasting aspects.

\subsection{Discussion}

The cross-tabulation revealed interesting clusters for the selected systems. For application domain, a cluster for health and comfort emerged, emphasizing delivery of personalized information. Interestingly, these systems operated at very different domains, which is reflected as unique properties in the cross-tabulation. In the cluster for improved security, the systems shared a strong emphasis for targeting the services for authorities.

The cross-tabulation for functionality revealed a cluster for decision making, where the systems shared the property of delayed response. The system in this cluster differed mostly on having maintenance or not.

The cross-tabulation for architecture revealed a cluster for open systems consisting, emphasizing particularly the use of open interfaces. The architectural similarity in this class could be explained by the fact that the systems are targeted for use by citizens. In the cluster for externally dependent systems, the cross-tabulation clearly reveals how the systems are built for different purposes. In this cluster, the combination of systems sharing some specific property varies substantially from property to property.

\section{Conclusion}

The classification method proposed here, produces relevant information about the considered systems, where clusters highlight interests shared by the systems and, at the same time, indicate how such systems differ from each other. Thus, The method is an excellent tool, when choosing a system from among several candidates. It is also a good tool for mapping competition, and strengthening an existing system, for instance, by emphasizing differentiating features.

One interesting topic for future research is to apply the classification method to understand qualitative properties of environmental monitoring systems, such as interoperability, reliability, robustness, and resilience. For such investigation, however, it would be necessary to involve the system developers, as required 
details tend not to be publicly available. For instance, interoperability seems to be a term easily used for any systems offering some external interfaces. However, as the clustering result in this article with respect to functionality viewpoint indicates, external dependability is not an unambiguous measure.

Acknowledgements. We wish to thank the Finnish Funding Agency for Technology and Innovation (TEKES) for funding this research. This work has been carried out in TEKES funded CLEEN SHOK programme, "Measurement, Monitoring and environmental Assessment" (MMEA). We wish also to thank CLEEN Ltd. and all our partners in MMEA for collaboration.

\section{References}

1. Messer, H., Zinevich, A., Alpert, P.: Environmental monitoring by wireless communication networks. Science 312, 713 (2006)

2. Cyranoski, D., Brumfiel, G.: Fukushima impact is still hazy. Nature 477, 139-140 (2011)

3. Gilbert, N.: Drug waste harms fish. Nature 476, 265 (2011)

4. Harlan, J., Terrill, E., Burnett, B.: National high frequency radar network: Update. In: OCEANS 2009, MTS/IEEE Biloxi - Marine Technology for Our Future: Global and Local Challenges. IEEE (2009)

5. Williams, W., Cornford, D., Ingram, B., Bastin, L., Beaumont, T., Pebesma, E., Dubois, G.: Supporting interoperable interpolation: the intamap approach. In: Swayne, D., Hrebicek, J. (eds.) Proceedings of International Symposium on Environmental Software Systems (2007)

6. Williams, M., Cornford, D., Bastin, L., Jones, R., Parker, S.: Automatic processing, quality assurance and serving of real-time weather data. Computers \& Geosciences 37, 351-362 (2011)

7. Williams, M., Cornford, D., Bastin, L., Pebesma, E.: Uncertainty markup language (UncertML). OpenGIS Discussion Paper 08-122r2. Open Geospatial Consortium Inc. (2009)

8. Skön, J., Kauhanen, O., Kolehmainen, M.: Energy consumption and air quality monitoring system. In: Proceedings of the 7th International Conference on Intelligent Sensors, Sensor Networks and Information Processing, pp. 163-167 (2011)

9. Rönkkö, M., Kolehmainen, M.: Titimake final report. In: Rönkkö, M., Kolehmainen, M. (eds.) University of Eastern Finland (2011)

10. Berlund, R., Kotovirta, V., Sein, A.: A system for icebreaker navigation and assistance planning using spaceborne sar information in the baltic sea. Canadian Journal of Remote Sensing 33(5), 378-387 (2007)

11. Kotovirta, V., Toivanen, T., Tergujeff, R., Huttunen, M.: Participatory sensing in environmental monitoring experiences. In: The Sixth International Conference on Innovative Mobile and Internet Services in Ubiquitous Computing, IMIS 2012 (2012) (accepted for publication) 